\title{
Riqueza de espécies de fungos conidiais em duas áreas de Mata Atlântica no Morro da Pioneira, Serra da Jibóia, BA, Brasil ${ }^{1}$
}

\author{
Marcos Fabio Oliveira Marques ${ }^{2,5}$, Luis Fernando Pascholati Gusmão ${ }^{3}$ e Leonor Costa Maia ${ }^{4}$
}

\author{
Recebido em 23/04/2007. Aceito em 31/01/2008
}

\begin{abstract}
RESUMO - (Riqueza de espécies de fungos conidiais em duas áreas de Mata Atlântica no Morro da Pioneira, Serra da Jibóia, BA, Brasil). A Serra da Jibóia, fragmento de Mata Atlântica situado no município de Santa Terezinha, Bahia, está incluído entre as áreas prioritárias para conservação. Com o objetivo de conhecer os fungos conidiais decompositores da serapilheira nesse ecossistema, foram realizadas cinco expedições a cada dois meses, de outubro/2005 a junho/2006. Trinta amostras de material vegetal (folhas, pecíolos, galhos e cascas) foram coletadas em três parcelas de $10 \mathrm{~m}^{2}$, eqüidistantes entre si $(10 \mathrm{~m})$, delimitadas em duas áreas com vegetação e umidade diferentes. As amostras foram lavadas em água corrente e incubadas em câmaras-úmidas por um mês; durante esse período as estruturas fúngicas foram retiradas dos substratos para estudo, sendo identificadas 106 espécies de fungos conidiais. Embora apresentando número equivalente de espécies, houve baixa similaridade (25\%), segundo o índice de Sørensen, entre as comunidades de fungos conidiais das duas áreas. Os dados contribuem para ampliar o conhecimento sobre a distribuição e a diversidade de fungos conidiais que colonizam substratos vegetais na Mata Atlântica e mostram a riqueza de espécies das áreas estudadas.
\end{abstract}

Palavras-chave: diversidade, Hyphomycetes, serapilheira

ABSTRACT - (Species richness of conidial fungi in two areas of Atlantic Forest at Morro da Pioneira, Serra da Jibóia, Bahia State, Brazil). The fragment of Atlantic Forest at Serra da Jibóia, municipality of Santa Terezinha, Bahia state, is a priority area for conservation. Five expeditions were undertaken every two months, from October/2005 to June/2006, in order to investigate the fungi that decompose leaf litter in this ecosystem. Thirty samples of plant debris (leaves, petioles, twigs and bark) were collected in three parcels of $10 \mathrm{~m}^{2}$, $10 \mathrm{~m}$ from each other, in two areas with different vegetation and humidity. Samples were washed in tap water and incubated in moist chambers for 30 days; during this period fungal structures on substrates were studied and 106 species of conidial fungi were identified. In spite of the similar number of species, there was low similarity $(25 \%)$ between the communities of conidial fungi of the two areas according to Sørensen's Index. These data increase our knowledge regarding the distribution and diversity of conidial fungi that colonize plant debris in the Atlantic Forest and confirm species richness in the areas studied.

Key words: diversity, Hyphomycetes, leaf litter

\section{Introdução}

A Floresta Atlântica guarda, apesar de séculos de destruição, a maior biodiversidade por hectare entre as Florestas Tropicais (Conti \& Furlan 2003). A fragmentação desse ecossistema tem conseqüências sobre a estrutura e os processos das comunidades vegetais, e as respostas dessa e de cada espécie ao habitat variam de acordo com fatores como histórico, tamanho e forma do fragmento, impacto das ações humanas, entre outras (Scariot et al. 2003). Nesses fragmentos de Mata Atlântica a grande quantidade de matéria orgânica produzida garante fertilidade suficiente para suprir a vegetação (Conti \& Furlan 2003). Associados à matéria orgânica, encontram-se fungos que garantem, junto com outros organismos, a ciclagem de nutrientes, disponibilizando-os aos vegetais e promovendo a manutenção desse ecossistema. Tais fungos exibem grande variedade morfológica e adaptativa a esse tipo de ambiente, favorecidos pelas condições adequadas de temperatura e umidade. Entretanto, o conhecimento sobre esses organismos ainda é escasso. Heredia et al. (1997) acreditam que, pela ampla diversidade de espécies que habitam os ecossistemas tropicais, é de se esperar que,

\footnotetext{
1 Parte da Dissertação de Mestrado do primeiro Autor, Programa de Pós-Graduação em Biologia de Fungos da Universidade Federal de Pernambuco, Brasil

2 Universidade do Estado da Bahia, Departamento de Educação, Campus VII, Laboratório de Microbiologia, BR 407, km 127, Rod. Lomanto Júnior, 48970-000 Senhor do Bonfim, BA, Brasil

3 Universidade Estadual de Feira de Santana, Departamento de Ciências Biológicas, Laboratório de Micologia, C. Postal 252, 44031-460 Feira de Santana, BA, Brasil

4 Universidade Federal de Pernambuco, Centro de Ciências Biológicas, Departamento de Micologia. Rua Professor Nelson Chaves s.n., 50670-420 Recife, PE, Brasil

5 Autor para correspondência: mfomarques@gmail.com
} 
exista riqueza considerável de fungos conidiais, habitantes típicos de substratos vegetais em decomposição.

A Serra da Jibóia representa um fragmento de Mata Atlântica, sendo uma área reconhecida como de extrema importância biológica no quesito vegetação (Brasil MMA 2002). Investigações sobre fungos nesse fragmento começaram em 1999 com estudos de espécies macroscópicas de Basidiomycota. No final do levantamento, Góes Neto et al. (2003) registraram 26 espécies distribuídas em 18 gêneros da ordem Aphyllophorales. Em relação a fungos decompositores da serapilheira da Serra da Jibóia, apenas dois trabalhos foram publicados Marques et al. (2007) que registraram 13 novas assinalamentos para América do Sul e descreveram uma nova espécie (Cubasina microspora M.F.O. Marques, Gusmão \& R.F. Castañeda) e Barbosa et al. (2007) catalogando 16 novas ocorrências de fungos conidiais para o neotrópico e duas novas espécies (Deightoniella rugosa F.R. Barbosa, Gusmão \& R.F. Castañeda e Diplocadiella cornitumida F.R. Barbosa, Gusmão \& R.F. Castañeda).

Neste trabalho são apresentados aspectos relacionados à comunidade de fungos conidiais associados à decomposição de folhas, pecíolos, galhas e cascas, coletados em duas áreas de mata na Serra da Jibóia, Bahia.

\section{Material e métodos}

A Serra da Jibóia, no município de Santa Terezinha, Bahia, é constituída por um complexo de morros com aproximadamente 5.928 ha, com altitude variando entre $750-840 \mathrm{~m}$, temperatura média anual de $21^{\circ} \mathrm{C}$, índice pluviométrico de $1.200 \mathrm{~mm} / \mathrm{ano}$ e chuvas concentradas de abril a julho, (Queiroz et al. 1996; M.A. Tomasoni, dados não publicados).

Foram realizadas cinco expedições, nos meses de outubro/dezembro/2005 e fevereiro/abril/junho/2006, em duas áreas do Morro da Pioneira (125'S e 39²8'W), na mencionada Serra. Uma constituída por mata fechada (Área A) com serapilheira profunda, $20-40 \mathrm{~cm}$, na vertente posterior de campo rupestre, umidade e temperatura variando de $70-95 \%$ e $19-24{ }^{\circ} \mathrm{C}$, respectivamente. Outra, constituída por mata com árvores esparsas (Área B), solo com Bromeliaceae e serapilheira com até $15 \mathrm{~cm}$ de profundidade, que antecede a formação do campo rupestre no topo da Serra, umidade e temperatura variando de $63-78 \%$ e $23-28{ }^{\circ} \mathrm{C}$, respectivamente.

Para estudo dos fungos conidiais foi realizada amostragem em três parcelas de $10 \mathrm{~m}^{2} \mathrm{em}$ cada área. As parcelas foram definidas a partir da borda da mata com $10 \mathrm{~m}$ de distância entre si, e em cada uma foram coletados aleatoriamente pecíolos, folhas, galhos e cascas (quatro subamostras de cada tipo de substrato) em vários estágios de decomposição, compondo uma amostra mista. No total foram coletadas, em cada expedição, seis amostras (três em cada área, contendo 12 subamostras com os diferentes substratos por área) resultando no final do estudo em 30 amostras (15 por área) de serapilheira nas diferentes áreas, as quais foram analisadas separadamente, para fins de comparação entre os substratos e áreas pesquisadas. Os fungos conidiais foram registrados pela análise de ocorrência (presença/ ausência) nos substratos.

As amostras foram submetidas à lavagem em água corrente, secagem sobre papel toalha em temperatura ambiente e posteriormente colocadas em câmaras-úmidas (placa de Petri + papel filtro), que foram acondicionadas em uma caixa de isopor $(170 \mathrm{~L})$. Periodicamente a caixa era aberta por alguns minutos para circulação do ar e após 72 horas o material incubado foi observado em estereomicroscópio. Durante 30 dias as estruturas reprodutivas dos fungos foram isoladas do material com auxílio de uma agulha fina e transferidas para meio de montagem entre lâmina e lamínula (Castañeda-Ruiz et al. 2006, modificado).

Para a identificação dos fungos foi utilizada bibliografia específica para cada gênero. O material estudado foi depositado no Herbário da Universidade Estadual de Feira de Santana (HUEFS) e/ou Herbário da Universidade Federal de Pernambuco, Departamento de Micologia (URM).

As espécies catalogadas foram agrupadas em categorias de constância utilizando-se o índice $\mathrm{C}=\mathrm{P} / \mathrm{N}$ $\times 100$, onde $\mathrm{P}=$ número de coletas contendo a espécie $\mathrm{e}$ $\mathrm{N}=$ número total de coletas, sendo considerado como táxon constante $(\mathrm{C})$ aquele cuja presença foi $\geq 50 \%$ nas amostras, como acessória (A) entre $\geq 25 \%$ e $<50 \%$ e acidental (S) quando < 25\% (Dajoz 1983).

A riqueza foi determinada pelo número total de espécies amostradas em cada área (Brower et al. 1998). A similaridade entre as áreas foi obtida a partir do coeficiente de Sørensen (Muller-Dombois 1981): IS $=2 \mathrm{c} / \mathrm{A}+\mathrm{B} \times 100$, onde $\mathrm{A}$ e $\mathrm{B}$ representam o número de espécies nas áreas A e B; c, o número de espécies comuns nas duas áreas.

\section{Resultados e discussão}

Foram identificados 106 táxons de fungos conidiais distribuídos em 71 gêneros, associadas a folhas, pecíolos, galhos e cascas. A micota na Área A apresentou 75 táxons, distribuídos em 50 gêneros, enquanto que na Área B foram registrados 69 táxons e 54 gêneros. Trinta e oito táxons foram comuns às duas áreas (Tab. 1).

$\mathrm{O}$ elevado número de fungos conidiais registrados no presente estudo, associados a substratos vegetais é 
Tabela 1. Fungos conidiais encontrados em duas áreas do Morro da Pioneira, Serra da Jibóia, município de Santa Terezinha, BA, Brasil, com os respectivos dados em relação ao substrato e categorias de constância. Substratos: Fs $=$ Folhas, Ps $=$ Pecíolos, Gs $=$ Galhos, Cs $=$ Cascas; Constância: $\mathrm{S}=$ Acidental, $\mathrm{A}=$ Acessória, $\mathrm{C}=$ constante.

\begin{tabular}{|c|c|c|c|c|c|c|c|c|c|}
\hline \multirow[t]{3}{*}{ Espécies } & \multicolumn{4}{|c|}{ Área A } & \multicolumn{4}{|c|}{ Área B } & \multirow[t]{3}{*}{ Categorias } \\
\hline & \multicolumn{8}{|c|}{ Substratos } & \\
\hline & Fs & Ps & Gs & $\overline{C s}$ & Fs & Ps & Gs & $\mathrm{Cs}$ & \\
\hline
\end{tabular}

Acrogenospora gigantospora S. Hughes

A. sphaerocephala (Berk. \& Broome) M.B. Ellis

Actinocladium rhodosporum Ehrenb.

Ardhachandra cristaspora (Matsush.) Subram. \& Sudha

Atrosetaphiale flagelliformis Matsush.

Beltrania africana S. Hughes

B. querna Harkn.

B. rhombica Penz.

Beltraniella portoricensis (F. Stevens) Piroz. \& S.D. Patil

Beltraniopsis esenbeckiae Bat. \& J.L. Bezerra

B. ramosa R.F. Castañeda

Brachysporiella gayana Bat.

Cacumisporium sigmoideum Mercado \& R.F. Castañeda

Canalisporium caribense (Hol.-Jech. \& Mercado) Nawawi \& Kuthub.

Ceratosporella compacta R.F. Castañeda, Guarro \& Cano

Chaetopsina fulva Rambelli,

Chalara alabamensis E.B.G. Jones \& E.G. Ingram

Chloridium lignicola (F. Mangenot) W. Gams \& Hol.-Jech.

Circinotrichum falcatisporum Piroz.

C. olivaceum (Speg.) Piroz.

Cladosporium sp.

Cryptophiale guadalcanalensis Matsush.

C. kakombensis Piroz.

C. udagawae Piroz. \& Ichinoe

Cryptophialoidea fasciculata Kuthub. \& Nawawi

Cubasina albofusca R.F. Castañeda

Dactylaria botulispora R.F. Castañeda \& W.B. Kendr.

Dendryphiopsis atra (Corda) S. Hughes

Dictyochaeta fertilis (S. Hughes \& W.B. Kendr.) Hol.-Jech.

D. nova-gieneensis (Matsush.) A.I. Romero

D. plurigutullata Kuthub. \& Nawawi

D. simplex (S. Hughes \& W.B. Kendr.) Hol.-Jech.

Dictyosporium digitatum J.L. Chen, C.H. Hwang \& Tzean

D. elegans Corda

Diplococcium stoveri (M.B. Ellis) R.C. Sinclair, Eicker \& Bhat

Ellisembia adscendens (Berk.) Subram.

Endophragmiella quadrilocularis Matsush.

Exserticlava triseptata (Matsush.) S. Hughes

E. vasiformis (Matsush.) S. Hughes

Gangliostilbe costaricensis Mercado, Gené \& Guarro

Gonytrichum macrocladum (Sacc.) Hughes

Gyrothrix magica Lunghini \& Onofri

G. microsperma (Höhn.) Piroz.

G. verticiclada (Goid.) S. Hughes \& Piroz.

Helicosporium sp.

Idriella ramosa Matsush.

Juenangia globulosa (Tóth) W.A. Baker \& Morgan-Jones

Kionochaeta nanophora Kuthub. \& Nawawi

Kionochaeta pughii Kuthub. \& Nawawi

K. ramifera (Matsush.) P.M. Kirk \& B. Sutton

K. spissa P.M. Kirk \& B. Sutton

Kylindria pluriseptata R.F. Castañeda

Lauriomyces heliocephalus (V. Rao \& de Hoog) R.F. Castañeda \&

W.B. Kendr.

Melanocephala australiensis (G.W. Beaton \& M.B. Ellis) S. Hughes

Monotosporella setosa var. setosa (Berk. \& M.A. Curtis) S. Hughes

\begin{tabular}{|c|c|c|c|c|c|c|c|c|}
\hline & & & $\mathrm{x}$ & & & & & $S$ \\
\hline & & & $x$ & & & & & A \\
\hline & & & & & & & $\mathrm{x}$ & A \\
\hline $\mathrm{X}$ & & & & & $\mathrm{X}$ & & & $\mathrm{A}$ \\
\hline X & & & & & $\mathrm{x}$ & & & $\mathrm{A}$ \\
\hline & $\mathrm{x}$ & & & & $\mathrm{x}$ & & & A \\
\hline $\mathrm{X}$ & $\mathrm{x}$ & & & $\mathrm{x}$ & $\mathrm{x}$ & & & C \\
\hline $\mathrm{x}$ & $\mathrm{x}$ & & & $\mathrm{x}$ & $\mathrm{x}$ & & & $\mathrm{C}$ \\
\hline $\mathrm{x}$ & $\mathrm{x}$ & & & $\mathrm{x}$ & $\mathrm{x}$ & & & C \\
\hline & & & & $\mathrm{x}$ & & & & A \\
\hline $\mathrm{X}$ & $\mathrm{x}$ & & & $\mathrm{x}$ & & & & C \\
\hline & & $\mathrm{x}$ & $\mathrm{X}$ & & & $\mathrm{x}$ & & C \\
\hline & & & & & & & $\mathrm{x}$ & $\mathrm{S}$ \\
\hline & & & $\mathrm{x}$ & & & & $\mathrm{x}$ & A \\
\hline $\mathrm{X}$ & $\mathrm{x}$ & & & & & & & A \\
\hline $\mathrm{x}$ & $\mathrm{x}$ & & & $\mathrm{x}$ & & & & $\mathrm{C}$ \\
\hline $\mathrm{x}$ & $\mathrm{x}$ & & $\mathrm{x}$ & & & & & C \\
\hline & & & & & & $\mathrm{x}$ & $\mathrm{x}$ & C \\
\hline $\mathrm{X}$ & & & & & & & & S \\
\hline $\mathrm{x}$ & & & & & & & & A \\
\hline & & & $\mathrm{x}$ & & & & & $S$ \\
\hline $\mathrm{X}$ & & & & & & & & $S$ \\
\hline $\mathrm{x}$ & & & & $\mathrm{x}$ & $\mathrm{x}$ & & & C \\
\hline & & & & $\mathrm{x}$ & & & & A \\
\hline & & & $\mathrm{x}$ & $\mathrm{x}$ & & & $\mathrm{x}$ & C \\
\hline & & & & & $\mathrm{x}$ & & & $S$ \\
\hline & & & & & & $\mathrm{x}$ & $\mathrm{x}$ & A \\
\hline & & & & & & $\mathrm{x}$ & & S \\
\hline $\mathrm{X}$ & & & & & & & & A \\
\hline & & $\mathrm{x}$ & & & & & & A \\
\hline & & $\mathrm{x}$ & & & & & & $S$ \\
\hline & $\mathrm{x}$ & & & $\mathrm{x}$ & & & & C \\
\hline & & & $\mathrm{x}$ & & & & & S \\
\hline & & & & & & & $\mathrm{x}$ & $S$ \\
\hline & $\mathrm{x}$ & & & & $\mathrm{x}$ & & & A \\
\hline & & $\mathrm{x}$ & $\mathrm{x}$ & & & & & C \\
\hline & & & & & $\mathrm{x}$ & & & $S$ \\
\hline & & & $\mathrm{x}$ & & & & $\mathrm{x}$ & $\mathrm{S}$ \\
\hline X & $\mathrm{X}$ & $\mathrm{x}$ & $\mathrm{x}$ & & $\mathrm{X}$ & $\mathrm{x}$ & $\mathrm{x}$ & $\mathrm{C}$ \\
\hline & & & $\mathrm{x}$ & & & & & $\mathrm{C}$ \\
\hline & & $\mathrm{x}$ & $\mathrm{x}$ & & & & $\mathrm{x}$ & $S$ \\
\hline$x$ & & & & & & & & $\mathrm{~A}$ \\
\hline $\mathrm{x}$ & & & & $\mathrm{x}$ & & & & C \\
\hline & & & & $\mathrm{x}$ & & & & C \\
\hline & $\mathrm{x}$ & & $\mathrm{x}$ & & & & & $S$ \\
\hline & & & & $\mathrm{x}$ & & & & S \\
\hline & & $\mathrm{x}$ & & & & $\mathrm{x}$ & & $\mathrm{C}$ \\
\hline & & $\mathrm{x}$ & & & & & & S \\
\hline & & $\mathrm{x}$ & & & & & & $S$ \\
\hline $\mathrm{X}$ & & & $\mathrm{x}$ & & & & $\mathrm{x}$ & $\mathrm{C}$ \\
\hline $\mathrm{x}$ & & & $\mathrm{x}$ & $\mathrm{x}$ & & & $x$ & $\mathrm{C}$ \\
\hline $\mathrm{x}$ & $\mathrm{x}$ & & & & & & & $\mathrm{C}$ \\
\hline & & & & & $\mathrm{x}$ & & & S \\
\hline & & & & & & & $\mathrm{x}$ & $S$ \\
\hline & & $\mathrm{x}$ & $\mathrm{x}$ & & & & & C \\
\hline
\end{tabular}


Tabela (continuação)

\begin{tabular}{|c|c|c|c|c|c|c|c|c|c|}
\hline \multirow[t]{3}{*}{ Espécies } & \multicolumn{4}{|c|}{ Área A } & \multicolumn{4}{|c|}{ Área B } & \multirow[t]{3}{*}{ Categorias } \\
\hline & \multicolumn{8}{|c|}{ Substratos } & \\
\hline & Fs & Ps & Gs & $\mathrm{Cs}$ & Fs & Ps & Gs & Cs & \\
\hline Nakataea fusispora (Matsush.) Matsush. & & & $\mathrm{x}$ & & & & $\mathrm{x}$ & & A \\
\hline Oidiodendron cerealis (Thüm.) G.L. Barron & & & $\mathrm{x}$ & & & & & & A \\
\hline Paraceratocladium polysetosum R.F. Castañeda & $\mathrm{x}$ & $\mathrm{x}$ & & & & & & & $\mathrm{C}$ \\
\hline P. silvestre R.F. Castañeda & $\mathrm{x}$ & & & & $\mathrm{X}$ & & & & $\mathrm{C}$ \\
\hline Parapleurotheciopsis inaequiseptata (Matsush.) P.M. Kirk & $\mathrm{x}$ & $\mathrm{x}$ & & & $\mathrm{x}$ & & & & $\mathrm{C}$ \\
\hline Parassympodiella laxa (Subram. \& Vittal) Ponnappa & $\mathrm{x}$ & $\mathrm{x}$ & & & $\mathrm{x}$ & $\mathrm{x}$ & & & $\mathrm{C}$ \\
\hline Periconia sp. & & & & & & $\mathrm{x}$ & & & $\mathrm{S}$ \\
\hline Phaeoisaria clematidis (Fuckel) S. Hughes & & & $\mathrm{x}$ & & & & $\mathrm{x}$ & & A \\
\hline P. infrafertilis B. Sutton \& Hodges & $\mathrm{x}$ & & & & & $\mathrm{x}$ & & & A \\
\hline Phialocephala humicola S.C. Jong \& E.E. Davis & & & & & & & & $\mathrm{x}$ & $\mathrm{S}$ \\
\hline Phragmocephala elegans R.F. Castañeda & & & & & $\mathrm{x}$ & $\mathrm{x}$ & & & A \\
\hline P. stemphylioides (Corda) S. Hughes & & $\mathrm{x}$ & & & & & & & $\mathrm{S}$ \\
\hline Pleurophragmium sp. & & & & & & & $\mathrm{x}$ & & $\mathrm{S}$ \\
\hline Pleurothecium recurvatum (Morgan) Höhn. & & & & $\mathrm{x}$ & & & & & $\mathrm{S}$ \\
\hline Pseudoacrodictys deightonii (M.B. Ellis) W.A. Baker \& Morgan-Jones & & & & & $\mathrm{x}$ & & & & $\mathrm{S}$ \\
\hline Pseudobotrytis terrestris (Timonin) Subram. & & $\mathrm{X}$ & & & & & $\mathrm{x}$ & & A \\
\hline Repetophragma fasciata $\mathrm{F}$. Castañeda, Gusmão \& Heredia & & & & & $\mathrm{X}$ & & & & $\mathrm{S}$ \\
\hline R. filiferum (Piroz.) R.F. Castañeda & & & & & & $\mathrm{x}$ & & & $\mathrm{S}$ \\
\hline Selenosporella curvispora G. Arnaud ex MacGarvie & $\mathrm{x}$ & $\mathrm{x}$ & & & $\mathrm{x}$ & $\mathrm{x}$ & & & A \\
\hline Speiropsis scopiformis Kuthub. \& Nawawi & & $\mathrm{x}$ & & & & $\mathrm{x}$ & & & $\mathrm{C}$ \\
\hline Sporidesmiella hyalosperma var. hyalosperma (Corda) P.M. Kirk & & $\mathrm{x}$ & & & & & $\mathrm{x}$ & $\mathrm{x}$ & $\mathrm{C}$ \\
\hline S. parva (M.B. Ellis) P.M. Kirk & & & $\mathrm{x}$ & & & & & & A \\
\hline S. vignalensis W.B. Kendr. \& R.F. Castañeda & & & & & $\mathrm{x}$ & & & & $\mathrm{S}$ \\
\hline Sporidesmium tropicale M.B. Ellis & & & $\mathrm{x}$ & & & & $\mathrm{x}$ & & A \\
\hline Stachybotrys bisbyi (Sriniv.) G.L. Barron & & $\mathrm{x}$ & & & & & & & $\mathrm{S}$ \\
\hline S. chartarum (Ehrenb.) S. Hughes & $\mathrm{x}$ & $\mathrm{x}$ & & & & & & & A \\
\hline S. kampalensis Hansf. & $\mathrm{x}$ & & & & & & & & $\mathrm{S}$ \\
\hline S. longispora Matsush. & & $\mathrm{X}$ & & & $\mathrm{x}$ & & & & A \\
\hline S. parvispora S. Hughes & $\mathrm{x}$ & $\mathrm{x}$ & & & & & & & A \\
\hline Stylaspergillus laxus B. Sutton, Alcorn \& P.J. Fisher & & & & & & $\mathrm{x}$ & & & $\mathrm{S}$ \\
\hline Subulispora longirostrata Nawawi \& Kuthub. & & & & & $\mathrm{x}$ & & & & $\mathrm{S}$ \\
\hline Thozetella cristata Piroz. \& Hodges & $\mathrm{x}$ & $\mathrm{x}$ & & & $\mathrm{x}$ & $\mathrm{x}$ & $\mathrm{x}$ & & $\mathrm{C}$ \\
\hline Thozetella sp. & & & & & & & $\mathrm{x}$ & & $\mathrm{S}$ \\
\hline Triposporium elegans Corda & & & $\mathrm{x}$ & & & & & & $\mathrm{S}$ \\
\hline Triposporium sp. & & $\mathrm{x}$ & & & & & & & $\mathrm{S}$ \\
\hline Umbellidion radulans B. Sutton \& Hodges & $\mathrm{x}$ & $\mathrm{x}$ & & & $\mathrm{x}$ & & & & $\mathrm{C}$ \\
\hline $\begin{array}{l}\text { Vermiculariopsiella cornuta (V. Rao \& de Hoog) Nawawi, } \\
\text { Kuthub. \& Sutton }\end{array}$ & & $\mathrm{x}$ & & & $\mathrm{x}$ & $\mathrm{x}$ & $\mathrm{x}$ & & $\mathrm{C}$ \\
\hline V. cubensis (Castañeda) Nawawi, Kuthub. \& B. Sutton & & & & & $\mathrm{x}$ & & & $\mathrm{S}$ & \\
\hline V. falcata Nawawi, Kuthub. \& B. Sutton & $\mathrm{x}$ & & & & & & & & $\mathrm{S}$ \\
\hline V. immersa (Desm.) Bender & & $\mathrm{x}$ & & & $\mathrm{x}$ & $\mathrm{x}$ & & & $\mathrm{C}$ \\
\hline Verticillium sp. & & & & & $\mathrm{x}$ & & & & $\mathrm{S}$ \\
\hline Virgaria nigra (Link) Nees & & & $\mathrm{x}$ & & & & & & $\mathrm{S}$ \\
\hline Virgatospora echinofibrosa Finley & & & & & & & $\mathrm{x}$ & & $\mathrm{S}$ \\
\hline Volutella minima Höhn. & $\mathrm{x}$ & $\mathrm{x}$ & & & & & & & $\mathrm{C}$ \\
\hline Volutella sp. & & & $\mathrm{x}$ & & & & & & $\mathrm{S}$ \\
\hline Wiesneriomyces laurinus (Tassi) P.M. Kirk & $\mathrm{x}$ & $\mathrm{x}$ & & & $\mathrm{x}$ & & & & $\mathrm{C}$ \\
\hline Yinmingella mitriformis Goh, K.M. Tsui \& K.D. Hyde & & & & & & & & $\mathrm{x}$ & $\mathrm{S}$ \\
\hline Zanclospora indica Subram. \& Vittal & & $\mathrm{x}$ & & & & & & & $\mathrm{S}$ \\
\hline Zanclospora novae-zelandiae S. Hughes \& W.B. Kendr. & & & & $\mathrm{x}$ & & & & & $\mathrm{S}$ \\
\hline Zygosporium echinosporum Bunting \& E.W. Mason & & & & & $\mathrm{x}$ & $\mathrm{x}$ & & & A \\
\hline Zygosporium gibbum (Sacc., M. Rousseau \& E. Bommer) S. Hughes & & & & & & $\mathrm{x}$ & & & $\mathrm{S}$ \\
\hline Total de ocorrências & 34 & 33 & 18 & 19 & 31 & 26 & 16 & 16 & - \\
\hline
\end{tabular}


evidenciado em outros estudos dessa natureza. Utilizando técnicas de observação direta e/ou indireta, vários trabalhos registraram alta riqueza de fungos conidiais associados a substratos vegetais. Bill \& Polishook (1994) obtiveram, em floresta úmida na Costa Rica, 1.709 isolados. Comparando microfungos isolados de folhas de Guarea guidonia (L.) Sleumer (Meliaceae) e Manilkara bidentata (A.D.C.) Chev. (Sapotaceae) em Porto Rico, Polishook et al. (1996) registraram 3.337 isolados, obtendo uma variação de 134 a 228 espécies/amostra. Os autores destacaram que Hyphomycetes e Coelomycetes foram os mais abundantes e, pelo índice de rarefação, observaram que amostras de $M$. bidentata foram relativamente uniformes na riqueza de espécies. Em estudos sobre a diversidade de fungos em 13 tipos de folhas em decomposição numa floresta úmida, na Austrália, Parungao et al. (2002) catalogaram 57 táxons: 18 Ascomycetes e 39 fungos conidiais. Investigando o folhedo de 71 espécies de plantas na Costa do Marfim Rambelli et al. (2004) identificaram 184 espécies de fungos, grande parte Hyphomycetes, verificando que $39,7 \%$ eram associadas com um substrato e $20,1 \%$ a dois substratos, sendo as espécies consideradas $59,8 \%$ especializadas, $12,5 \%$ ubíquas e 27,7\% comuns.

Grande parte das espécies encontradas nas áreas de estudo foram também registradas em inventários realizados em outras áreas de Mata Atlântica, no sudeste do Brasil (Grandi 1998; 1999; 2004, Grandi \& Attili 1996, Grandi \& Gusmão 2002; Grandi \& Silva 2006; Gusmão \& Grandi 1997 e Gusmão et al. 2001).

Apesar dos diversos levantamentos não serem comparáveis, pois foram realizados em áreas e períodos diferentes, o folhedo de Caesalpinia echinata Lam. foi segundo Grandi \& Silva (2006) um dos mais investigados contribuindo para o conhecimento de fungos conidiais no Brasil. Por outro lado, os estudos ainda são incipientes em diversas áreas, como observado pela considerável riqueza taxonômica encontrada no fragmento de Mata Atlântica ora investigado.

A alta riqueza de espécies nos trópicos permite inferir que o estudo dessas plantas e do folhedo por elas produzido pode levar ao conhecimento de novos gêneros e espécies com caracteres singulares (Mercado-Sierra et al. 1987). Do mesmo modo, tem levado à descoberta de gêneros de fungos conidiais pouco estudados, alguns monotípicos como os encontrados nesse trabalho Atrosetaphiale, Cubasina, Stylaspergillus, Umbellidion e Yinmingella.

$\mathrm{Na}$ Área A, 14 espécies foram comuns às três parcelas amostradas: Beltrania rhombica, Beltraniella portoricensis, Brachysporiella gayana, Chalara alabamensis, Chaetopsina fulva, Exserticlava vasiformis, Gangliostilbe costaricensis, Kionochaeta ramifera, Monotosporella setosa var. setosa,
Paraceratocladium silvestre, Speiropsis scopiformis, Stachybotrys parvispora, Umbellidion radulans e Volutella minima, enquanto que na Área B apenas nove espécies ocorreram nas três parcelas: Beltrania rhombica, B. querna, Beltraniella portoricensis, Chaetopsina fulva, Cryptophiale kakombensis, Exserticlava vasiformis, Gyrothrix microsperma, Parassympodiella laxa e Vermiculariopsiella cornuta. $\mathrm{O}$ número de espécies exclusivas e comuns às três parcelas variou em cada área (Fig. 1). Essas diferenças refletem a heterogeneidade dos microhabitats nas áreas investigadas.
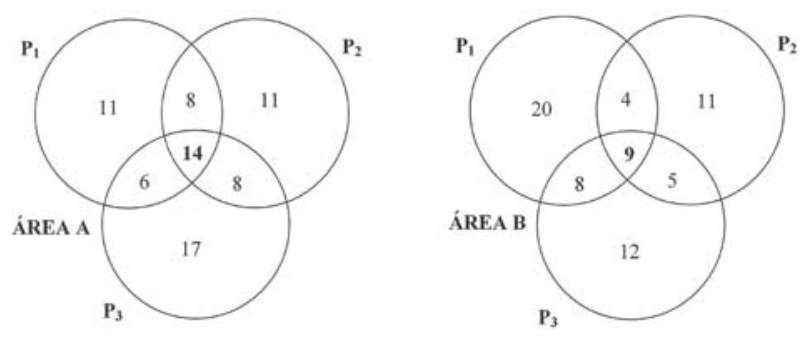

Figura 1. Espécies de fungos conidiais exclusivas e comuns às parcelas estudadas $\left(\mathrm{P}_{1}, \mathrm{P}_{2}, \mathrm{P}_{3}\right)$ nas áreas $\mathrm{A}$ e $\mathrm{B}$, associadas a folhas, pecíolos, galhos e cascas da serapilheira, na Serra da Jibóia, BA, Brasil.

Foram mais representativos os fungos conidiais associados à decomposição de folhas e pecíolos. Houve predominância, nesse caso, de espécies do complexoBeltrania (6), Stachybotrys (5), Vermiculariopsiella (4) e Cryptophiale (3). No caso dos associados a galhos e cascas, tiveram destaque Kionochaeta (4), Acrogenospora e Exserticlava (2) (Tab. 2). As folhas são mais susceptíveis aos fungos por representar a parte mais significativa entre os substratos vegetais da serapilheira, pela biomassa e pelo conteúdo de nutrientes orgânicos e inorgânicos que possuem (Meguro et al. 1979). Dix \& Webster (1995) destacaram que as folhas são um dos mais relevantes substratos colonizados por microrganismos, entre eles os fungos conidiais. Estudando a diversidade de fungos filamentosos em mangue, na Índia Ananda \& Sridhar (2004) evidenciaram, pela análise dos substratos (folhas, madeira e cascas), as preferências fúngicas e a variação durante o processo de sucessão, registrando grande diversidade e riqueza de espécies em madeira em decomposição (65) e folhedo (49). Esses resultados são diferentes do registrado no presente estudo, em área de mata, o que reflete as particularidades de cada ambiente e dos substratos estudados.

A distribuição das espécies por classes de constância (Tab. 2) revelou a predominância das acidentais $(42,5 \%)$, seguidas das constantes $(31,1 \%)$ e acessórias $(26,4 \%)$. Entre os fungos encontrados, apenas Exserticlava vasiformis foi observado colonizando todos os substratos. 
Tabela 2. Riqueza de gêneros e espécies de Fungos Conidiais registrados no fragmento de Mata Atlântica, Serra da Jibóia, BA, Brasil. $\mathrm{S}=$ Acidental, $\mathrm{A}=$ Acessória e $\mathrm{C}=$ Constante.

\begin{tabular}{|c|c|c|c|c|c|}
\hline \multirow[t]{2}{*}{ Fungos conidiais } & \multirow[t]{2}{*}{ Gêneros } & \multirow[t]{2}{*}{ Espécies } & \multicolumn{3}{|c|}{ Constância \% } \\
\hline & & & $\mathrm{S}$ & A & $\mathrm{C}$ \\
\hline Associados a folhas & 14 & 18 & 9,4 & 4,7 & 2,9 \\
\hline Associados a pecíolos & 15 & 15 & 11,5 & 1,9 & 0,9 \\
\hline Associados a galhos & 15 & 17 & 9,4 & 5,7 & 0,9 \\
\hline Associados a cascas & 13 & 15 & 10,4 & 2,9 & 0,9 \\
\hline Comuns a folhas e pecíolos & 22 & 25 & - & 9,4 & 14,2 \\
\hline Comuns a folhas e galhos & - & - & - & - & - \\
\hline Comuns a folhas e cascas & 2 & 3 & - & - & 2,9 \\
\hline Comuns a pecíolos e galhos & 1 & 1 & - & 0,9 & - \\
\hline Comuns a pecíolos e cascas & 1 & 1 & 0,9 & - & - \\
\hline Comuns a galhos e cascas & 6 & 6 & 0,9 & 0,9 & 3,8 \\
\hline Comuns a folhas, pecíolos e galhos & 2 & 2 & - & - & 1,9 \\
\hline Comuns a folhas, pecíolos e cascas & 1 & 1 & - & - & 0,9 \\
\hline Comuns a folhas, galhos e cascas & - & - & - & - & - \\
\hline Comuns a pecíolos, galhos e cascas & 1 & 1 & - & - & 0,9 \\
\hline Comuns a folhas, pecíolos, galhos e cascas & 1 & 1 & - & - & 0,9 \\
\hline Total & 78 & 106 & 42,5 & 26,4 & 31,1 \\
\hline
\end{tabular}

Associadas a folhas e pecíolos foram registradas 15 espécies constantes e 10 acessórias; no entanto, colonizando apenas pecíolos foram 12 espécies acidentais, com menor incidência de espécies acessórias (2) e constantes (1), seguidos do registro de espécies acidentais em cascas (11), folhas (10) e galhos (10) (Fig. 2).

Estudando a micota associada a três tipos de folhas em floresta no México, Heredia (1993) registrou a presença esporádica de muitos fungos durante o processo de sucessão, sendo poucas espécies constantes (Beltrania rhombica, Beltraniella portoricensis (como Ellisiopsis gallesiae), Codianea assamica, Chalara alabamensis, Cryptophiale kakombensis, Cylindrocladium scoparium e Subulispora procurvata) durante todo o processo. Bill \& Polishook (1994) registraram poucas espécies abundantes e uma alta proporção de espécies raras e entre os fungos

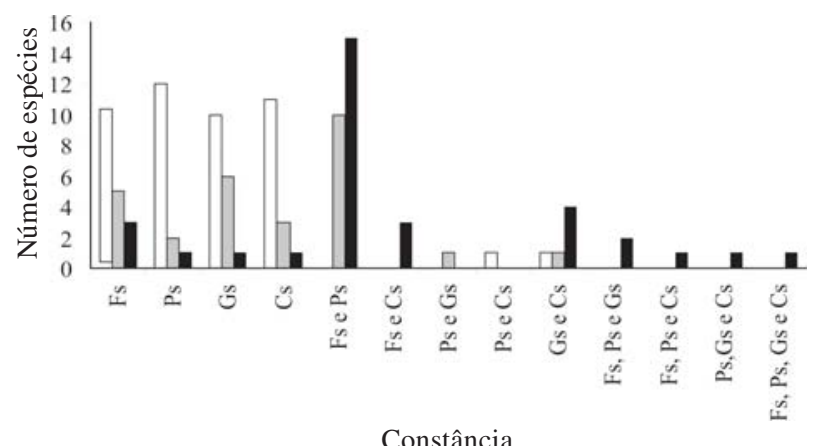

Figura 2. Número de espécies, considerando as categorias de constância em diferentes substratos em área de Mata Atlântica na Serra da Jibóia, BA, Brasil. Fs = Folhas, Ps = Pecíolos, Gs = Galhos, Cs = Cascas. $(\square=$ Acidental; $\square=$ Acessória; $\mathbf{\square}=$ Constante $)$. responsáveis pela decomposição destacaram Beltrania rhombica, Cryptophiale sp., Dictyochaeta sp., Volutella minima, entre outros também registrados nesse estudo. A freqüência de ocorrência dos fungos depende, entre outros fatores, das condições ambientais.

A similaridade de espécies de fungos conidiais entre as áreas estudadas pode ser considerada baixa (25\%). Esse resultado possivelmente decorre das diferenças das espécies vegetais e características ambientais entre as duas áreas investigadas e, segundo Polishook et al. (1996), amostras de folhedo de uma mesma espécie de planta tendem a ter alta similaridade, comparadas às amostras de diferentes espécies vegetais em uma mesma área. A vegetação do local estudado constitui um mosaico, com diferentes substratos passíveis de colonização pelos fungos. Na vertente oriental predomina a Floresta Ombrófila Densa, com remanescentes de Mata Atlântica, influenciada pelas chuvas orográficas onde os ventos úmidos, oriundos do litoral, ao encontrar a serra, fazem precipitar grande parte da umidade que contribui para manter sua densidade e exuberância. A porção ocidental é menos úmida, apresentando Floresta Estacional Semidecidual (onde foram realizadas as coletas), Floresta Decidual e Caatinga Arbórea com palmeiras, onde as plantas encontram-se espaçadas, apresentando baixa densidade (M.A.Tomasoni, dados não publicados).

A comparação da similaridade entre as populações de fungos conidiais nos substratos investigados mostrou grande variação (Tab. 3), indo de 3,0 (galhos $\times$ pecíolos) a 30,1 (folhas $\times$ pecíolos).

Heredia (1993) comparou a similaridade de fungos entre folhas de Quercus germana Cham. \& Schlrcht, 
Tabela 3. Matriz de semelhança a partir do índice de Sørensen, entre as diversidades de fungos conidiais identificados, nos substratos investigados.

\begin{tabular}{lcccc}
\hline Substratos & Folhas & Pecíolos & Galhos & Cascas \\
\hline Folhas & 100 & & & \\
Pecíolos & 30,1 & 100 & & \\
Galhos & 0,0 & 3,0 & 100 & \\
Cascas & 9,3 & 3,2 & 27,2 & 100 \\
\hline
\end{tabular}

Q. sartorii Liemb. e Liquidambar styraciflua L. observando alta similaridade entre elas (acima de 70\%). No Brasil, Maia (1983) verificou que as populações fúngicas em Licania octandra (Hoffmgg. ex R. \& S.) Kuntze e L. kunthiana Hook F. são mais semelhantes entre si $(69,99 \%)$, de que as populações em L. octandra e Hortia arborea Engl. (64,01\%) e em L. kunthiana e H. arbórea (63,45\%). Lodge (1997) e Lodge \& Cantrell (1995) sugerem comparações entre plantas de uma mesma espécie, considerando variáveis como o local e distúrbios ambientais, pois estes afetam a distribuição dos fungos conidiais colonizadores da serapilheira.

Polishook et al. (1996) argumentam que os fungos têm preferência por um determinado substrato, resultando na riqueza fúngica do folhedo misto florestal. Fato este também observado por Hyde \& Alias (2000) relatando que diferentes partes do vegetal (folhas, pecíolos, cascas, etc.) abrigam diferentes fungos, indicando que alguns deles podem preferencialmente se desenvolver em certos tipos de tecido, como as espécies endofíticas. No entanto, Parungao et al. (2002) acreditam que poucos fungos decompositores são específicos de um substrato. A partir dos valores de constância das espécies encontradas nesse estudo, observa-se que a maioria ocorre associada a apenas um substrato (Tab. 2).

Entre as espécies identificadas, algumas apresentam ampla distribuição: Beltrania rhombica, Beltraniella portoricensis (Matsushima 1971; 1975), Dictyochaeta simplex (Mercado-Sierra et al. 1997), Melanocephala australiensis (Hughes 1978), Pleurothecium recurvatum (Goos 1969), Sporidesmium tropicale (Mckenzie 1995), Stachybotrys chartarum (Mercado-Sierra et al. 1997), Virgaria nigra (Heredia et al. 1997), entre outros. Outras, no entanto, são escassamente referidas na literatura: Ceratosporella compacta (Castañeda-Ruiz et al. 1996), Dactylaria botulispora (Castañeda-Ruiz \& Kendrick 1990), Endophragmiella quadrilocularis (Matsushima 1993), Kionochaeta nanophora, Kionochaeta pughii (Kuthubutheen \& Nawawi 1988), Sporidesmiella vignalensis (Castañeda-Ruiz \& Kendrick 1990), Yimingiella mitriformis (Goh et al. 1998), Zanclospora novae-zelandiae (Hughes \& Kendrick 1965) entre outras. O predomínio de uma espécie está ligado aos fatores climáticos e físico-químicos dos substratos encontrados na área investigada. Outra explicação é que as técnicas aplicadas podem favorecer alguns fungos pelas condições nutricionais os quais são submetidos.

Espécies cosmopolitas e pantropicais como B. rhombica, B. portoricencis, Cladosporium cladosporioides, Colletotrichum gloeosporioides, Fusarium solani, Gliocladium roseum, Idriella lunata, Paecilomyces marquandii, Lasiodiplodia theobromae, Scolecobasidium constrictum, Mucor hiemalis, Phomopsis spp. Trichoderma spp., Volutella minima e Wiesneriomyces laurinus são usualmente isoladas durante estudos de folhedo de florestas tropicais úmidas e os gêneros Chaetopsina, Chloridium, Clonostachys, Cryptophiale, Dactylaria, Dictyochaeta, Myrothecium, Scolecobasidium, Stachybotrys e muitos anamorfos de Hypocreales e Xylariales estão representados na micota de folhedo tropical (Polishook et al. 1996).

O levantamento de fungos conidiais que colonizam substratos vegetais em decomposição tem sido eficaz para ampliar o conhecimento sobre a diversidade desses microrganismos, permitindo que novas espécies sejam conhecidas e novos registros sejam feitos, o que abre a possibilidade de sua posterior utilização para fins diversos. Como destacaram Heredia-Abarca et al. (2004), a importância dos fungos conidiais não se limita ao papel que desempenham no equilíbrio ecológico como degradadores de restos orgânicos e reguladores de populações fitopatogênicas

Os dados demonstram a elevada diversidade de fungos conidiais nas áreas estudadas. Considerando que o desmatamento dos remanescentes de Mata Atlântica no Brasil continua, entende-se como de fundamental importância conhecer a micota nesse bioma, de modo a fornecer subsídios para a conservação.

\section{Referências bibliográficas}

Ananda, K. \& Sridhar, K.R. 2004. Diversity of filamentous fungi on decomposing leaf litter of mangrove forests in the southwest coast of India. Current Science 87: 1431-1437.

Barbosa, F.R.; Gusmão, L.F.P.; Castañeda-Ruiz, R.F.; Marques, M.F.O.; \& Maia, L.C. 2007. Conidial fungi from the semi-arid Caatinga Biome of Brazil. New species Deightoniella rugosa \& Diplocladiella cornitumida with new records for the neotropics. Mycotaxon 102: 39-49.

Bill, G.F. \& Polishook, J.D. 1994. Abundance and diversity of microfungi in leaf litter of a lowland rain forest in Costa Rica. Mycologia 86: 187-198.

Brasil-MMA. 2002. Avaliação e ações prioritárias para a conservação da biodiversidade da Mata Atlântica e Campos Sulinos. Pp. 215-266. In: Avaliação e identificação de áreas e ações prioritárias para conservação e utilização sustentável e repartição dos benefícios da Biodiversidade nos Biomas Brasileiros. Brasília, MMA/SBF.

Brower, J.E.; Zar, J.H. \& Von Ende, C.A. 1998. Field and laboratory methods for general ecology. $4^{\text {th }}$ ed. Dubuque, Wm. C. Brown Publishers. 
Castañeda-Ruiz, R.F. 1985. Deuteromycotina de Cuba. Hyphomycetes III. Habana. Instituto de Investigaciones Fundamentales en agricultura Tropical "Alejandro de Humboldt".

Castañeda-Ruiz, R.F. \& Kendrick, B. 1990. Conidial Fungi from Cuba: II. University of Waterloo Biology series 3.

Castañeda-Ruiz, R.F.; Guarro, J. \& Cano, J. 1996. Notes on conidial fungi. X. A new species of Ceratosporella and some new combinations. Mycotaxon 60: 275-281.

Castañeda-Ruiz, R.F; Gusmão, L.F.P.; Heredia-Abarca, G. \& Saikawa, M. 2006. Some Hyphomycetes from Brazil. Two new species of Brachydesmiella. Two new combinations for Repetophragma, and new records. Mycotaxon 95: 261-270.

Conti, J.B. \& Furlan, S.A. 2003. Geoecologia: o clima, os solos e a biota. Pp. 67-237. In: J.L.S. Ross (org.). Geografia do Brasil São Paulo, EDUSP.

Dajoz, R. 1983. Ecologia Geral. Petrópolis, Vozes.

Dix, N.J. \& Webster, J. 1995. Fungal Ecology. London, Chapman \& Hall.

Góes Neto, A.; Marques, M.F.O.; Andrade, J.D. \& Santos, D.S. 2003. Lignicolous Aphyllophoroid Basidiomycota in an Atlantic Forest Fragment in the semi-arid caatinga region of Brazil. Mycotaxon 88: $359-364$.

Goh, T.K.; Tsui, K.M. \& Hyde, K.D. 1998. Yinmingella mitriformis gen. et sp. nov., a new sporodochial hyphomycete from submerged wood in Hong Kong. Mycological Research 76 1717-1724.

Goos, R.D. 1969. The genus Pleurothecium. Mycologia 61 1048-1053

Grandi, R.A.P. 1998. Hyphomycetes decompositores do folhedo de Alchornea triplinervia (Spreng.) Müll. Arg. Hoehnea 25 133-148.

Grandi, R.A.P. 1999. Hifomicetos decompositores do folhedo de Euterpe edulis Mart. Hoehnea 26: 87-101.

Grandi, R.A.P. 2004. Anamorfos da Serapilheira nos Vales dos Rios Moji e Pilões, município de Cubatão, São Paulo, Brasil. Hoehnea 31: 225-238.

Grandi, R.A.P. \& Attili, D.S. 1996. Hyphomycetes on Alchornea triplinervia (Spreng.) Müell. Arg. leaf litter from the Ecological Reserve Juréia-Itatins, State of São Paulo, Brazil. Mycotaxon 60: $373-386$.

Grandi, R.A.P. \& Gusmão, L.F.P. 2002. Hyphomycetes decompositores do folhedo de Tibouchina pulchra Cogn.. Revista Brasileira de Botânica 25: 79-87.

Grandi, R.A.P. \& Silva, T.V. 2006. Fungos anamorfos decompositores do folhedo de Caesalpinia echinata Lam. Revista Brasileira de Botânica 29: 275-287.

Gusmão, L.F.P. \& Grandi, R.A.P. 1997. Hyphomycetes com conidioma dos tipos esporodóquio e sinema associados à folhas de Cedrela fissilis (Meliaceae), em Maringá, PR, Brasil. Revista Brasileira de Botânica 11: 123-133.

Gusmão, L.F.P.; Grandi, R.A.P. \& Milanez, A.I. 2001. Hyphomycetes from leaf of Miconia cabussu in the Brazilian Atlântic rain forest. Mycotaxon 79: 201-213.

Heredia, G. 1993. Mycoflora associated with green leaves and leaf litter of Quercus germana, Quercus sartorii and Liquidambar styraciflua in a mexican cloud forest. Cryptogamie Mycologique 14: 171-183.

Heredia, G.; Mena-Portales, J. \& Mercado-Sierra, A. 1997. Hyphomycetes saprobios tropicales. Nuevos registros de Dematiáceos para Mexico. Revista del Jardim Botanico 13: 41-51.

Heredia-Abarca, G.; Estebanez, M.R.; Mota, R.M.A.; Mena-Portales, J. \& Mercado-Sierra, A. 2004. Adiciones al conocimiento de la diversidad de los hongos conidiales del Bosque Mesófilo de Montaña del estado de Veracruz. Acta Botanica Mexicana 66 $1-22$.
Hughes, S.J. 1978. New Zealand Fungi 25. Miscellaneous species. New Zealand Journal of Botany 16: 311-370.

Hughes, S.J. \& Kendrick, W.W. 1965. New Zeland Fungi 4. Zanclospora Gen. Nov. New Zealand Journal of Botany 3: $151-158$.

Hyde, K.D. \& Alias, S.A. 2000. Biodiversity and distribution of fungi associated with decomposing Nypa fruticans. Biodiversity and Conservation 9: 393-402.

Kuthubutheen, A.J. \& Nawawi, A. 1988. Two new species of Kionochaeta (Hyphomycetes) and K. ramifera from Maylasia. Transactions British Mycological Society 90: 165-177.

Lodge, D.J. 1997. Factors related to diversity of decomposer fungi in tropical forests. Biodiversity and Conservation 6: 681-688.

Lodge, D.J. \& Cantrell, S. 1995. Fungal communities in wet tropical forests: variation in time and space. Canadian Journal of Botany (suppl.) 73: 1391-1398.

Maia, L.C. 1983. Sucessão de fungos em folhedo de floresta tropical úmida. Recife, Ed. Universitária.

Marques, M.F.O.; Barbosa, F.R.; Gusmão, L.F.P.; Castañeda-Ruiz, R.F. \& Maia, L.C. 2007. Conidial fungi from the semi-arid Caatinga Biome of Brazil. Cubasina microspora sp. Nov., a note on $C$. albofusca, and some new records for Sout America. Mycotaxon 102: 17-23.

Matsushima, T. 1971. Microfungi of the Solomon islands and Papua-New Guinea. Published by the author. Kobe.

Matsushima, T. 1975. Icones Microfungorum a Matsushima Lectorum. Published by the author. Kobe.

Matsushima, T. 1993. Matsushima Mycological Memoirs n. 7. Published by the author. Kobe.

Mckenzie, E.H.C. 1995. Dematiaceous Hyphomycetes on Pandanaceae. 5. Sporidesmium sensu lato. Mycotaxon 56: 9-29.

Meguro, M.; Vinueza, G.N. \& Delitti, W.B.C. 1979. Ciclagem de nutrientes minerais na mata mesófila secundária - São Paulo. I Produção e conteúdo de nutrientes minerais no folhedo. Boletim de Botânica da Universidade de São Paulo 7: 11-31.

Mercado-Sierra, A.; Holubová-Jechova, V. \& Mena-Portales, J. 1997. Hifomicetos demaciáceos de Cuba, Enteroblásticos. Torino, Museo Regionale di Scienze Naturali, Monografie XIII.

Mercado-Sierra, A.; Holubová-Jechová, V.; Mena-Portales, J. \& Fraginals-Gonzàles, G. 1987. Hongos imperfectos de Pinar del Rio, Cuba: El ambiente y la taxonomia de hifomicetes demaciáceos hallados. Acta Botanica Cubana 22: 1-10.

Muller-Dombois, D. 1981. Ecological measurements and microbial populations. Pp. 173-184. In: D.T. Wicklow \& G.C. Carroll (eds.). The fungal community: Its organization and role in the ecosystem. New York, Marcel Derker.

Parungao, M.M.; Fryar, S.C. \& Hyde, K.D. 2002. Diversity of fungi on rainforest litter in North Queensland, Australia. Biodiversity and Conservation 11: 1185-1194.

Polishook, J.D.; Bills, G.F. \& Lodge, D.J. 1996. Microfungi from decaying leaves of two rain forest trees in Puerto Rico. Journal of Industrial Microbiology 17: 284-294.

Queiroz, L.P.; Sena, T.S.N. \& Costa, M.J.S.L. 1996. Flora vascular da Serra da Jibóia, Santa Terezinha - Bahia. I: O Campo Rupestre. Sitientibus 15: 27-40.

Rambelli, A.; Mulas, B. \& Pasqualetti, M. 2004. Comparative studies on microfungi in tropical ecosystems in Ivory Coast Forest litter: behaviour on different substrata. Mycological Research 108: $325-336$.

Scariot, A.; Freitas, S.R.; Mariano Neto, E.; Nascimento, M.T.; Oliveira, L.C.; Sanaiotti, T. \& Sevilha, A.C. 2003. Vegetação e flora. Pp. 103-126. In: D.M. Rambaldi \& D.A. Suaréz de Oliveira (orgs.). Fragmentação de Ecossistemas: causas, efeitos sobre a biodiversidade e recomendações de políticas públicas. Brasília, MMA/SBF. 